\title{
Transoesophageal echocardiography as the sole diagnostic investigation in patients with suspected thoracic aortic dissection
}

\author{
Adrian P Banning, Navroz D Masani, Shahid Ikram, Alan G Fraser, Roger J C Hall
}

\begin{abstract}
Objectives-To assess the value and limitations of using transoesophageal echocardiography as the sole diagnostic test in patients with suspected thoracic aortic dissection.
\end{abstract}

Design-Retrospective data review over a two year period.

Setting-A regional cardiothoracic centre. Patients-Data were compiled from admission records, surgical records, and lists of patients undergoing diagnostic investigations in the hospital. Patient's notes were used to identify presentation, management, and outcome.

Interventions-Patients were managed according to the policy of our unit, which is to treat patients with dissection affecting the ascending aorta by an operation. Patients with uncomplicated dissection sparing the ascending aorta are initially managed medically.

Main outcome measures-In hospital and two year follow up of patients who were investigated by transoesophageal echocardiography alone.

Results-Of 48 patients referred, 45 underwent transoesophageal echocardiography. Dissection was confirmed in 22 patients. Transoesophageal echocardiography showed the proximal extent of the dissection in $21 / 22(96 \%)$ and only one patient required a further diagnostic investigation. Ten patients with dissection of the ascending aorta underwent graft replacement of the ascending aorta; operative mortality was $10 \%$ and their two year survival was $80 \%$. Of the eight patients with dissection of the descending aorta, six were discharged home, and five were alive at two years. No patient without evidence of dissection on their initial transoesophageal echocardiographic examination required re-investigation into possible dissection in the two years after discharge.

Conclusions-In patients with suspected thoracic dissection transoesophageal echocardiography rapidly and safely gives all the necessary diagnostic information. Further investigations, including coronary angiography, before surgery are unnecessary.

(Br Heart f 1994;72:461-465)
Patients with suspected thoracic aortic dissection require rapid, safe, and accurate diagnostic imaging. ${ }^{1}$ In most centres, aortography via the femoral artery has been superseded by computed tomography, magnetic resonance imaging, or transoesophageal echocardiography, as these techniques are non-invasive and have greater sensitivity and specificity. ${ }^{2}$

In our unit transoesophageal echocardiography has been the primary diagnostic test for thoracic aortic dissection since 1990. It gives detailed diagnostic information, including data on the presence of aortic regurgitation, pericardial effusion, thrombus in the false lumen, and involvement of the coronary artery ostia, ${ }^{3}$ making complementary investigations such as angiography unnecessary. This approach minimises delay and allows rapid transfer to the operating theatre.

To assess the advantages and limitations of managing patients on the basis of echocardiographic findings alone we reviewed our experience over two years.

Patients and methods

The University Hospital of Wales is the regional cardiothoracic centre for Wales. The names of all patients referred with possible thoracic dissection between May 1990 and May 1992 were obtained retrospectively using admission records, surgical records, and lists of patients undergoing transoesophageal echocardiography, thoracic computed tomography, and aortography. Patients' notes were then used to gather details of presentation, management, and outcome. Follow up data were available on all patients included in the study and were obtained from hospital and general practitioner records.

Patients were admitted to the cardiac care unit where systolic blood pressure was maintained below $110 \mathrm{~mm} \mathrm{Hg}$ by intravenous infusions of sodium nitroprusside or labetalol, or both. Immediate transthoracic echocardiography was followed by transoesophageal echocardiography performed using a Hewlett Packard Sonos 1000 or 1500 ultrasound machine and a biplane (23 patients) or single plane (22 patients) $5 \mathrm{MHz}$ transoesophageal probe. Investigations were performed on the cardiac care unit by one of four experienced operators.

Patients were managed according to the policy of our unit, which is to treat patients 
with dissection affecting the ascending aorta (Stanford classification type $\mathrm{A})^{4}$ surgically. Patients with uncomplicated dissection sparing the ascending aorta (type B) are initially managed medically by aggressive control of blood pressure and prolonged bed rest. They are considered for surgical intervention if there is evidence of extension of the dissection, progressive aortic enlargement, or ischaemic complications.

\section{Results}

Forty eight patients (aged 24-84 years, median 67 years, 21 men) were referred with suspected acute aortic dissection. Transoesophageal echocardiography was performed in $45(94 \%)$ patients after intravenous sedation with midazolam $(2 \cdot 5-12.5 \mathrm{mg}$; mean 4 $\mathrm{mg}$ ). The procedure was tolerated by all patients and there was no significant complication. Three patients referred from district general hospitals with dissection diagnosed by computed tomography were considered too ill for either transoesophageal echocardiography or an operation. All three died shortly after admission; the diagnoses of dissection were confirmed at necropsy.

\section{FINDINGS OF TRANSOESOPHAGEAL \\ ECHOCARDIOGRAPHY}

Aortic dissection was confirmed in 22/45 (49\%) patients (aged 24-84 years, mean 61 years); 15 had type $A$ dissection and seven type B. Transoesophageal echocardiography (10 biplane, 12 monoplane) identified the proximal extent of the dissection in 21 (96\%) patients. In the remaining patient it showed dissection in the descending thoracic aorta and a linear shadow in the ascending aorta, which was thought to represent a dissection flap. Surgical inspection of the ascending aorta revealed annulo-aortic ectasia alone, with dissection of the descending aorta below the origin of the left subclavian artery.

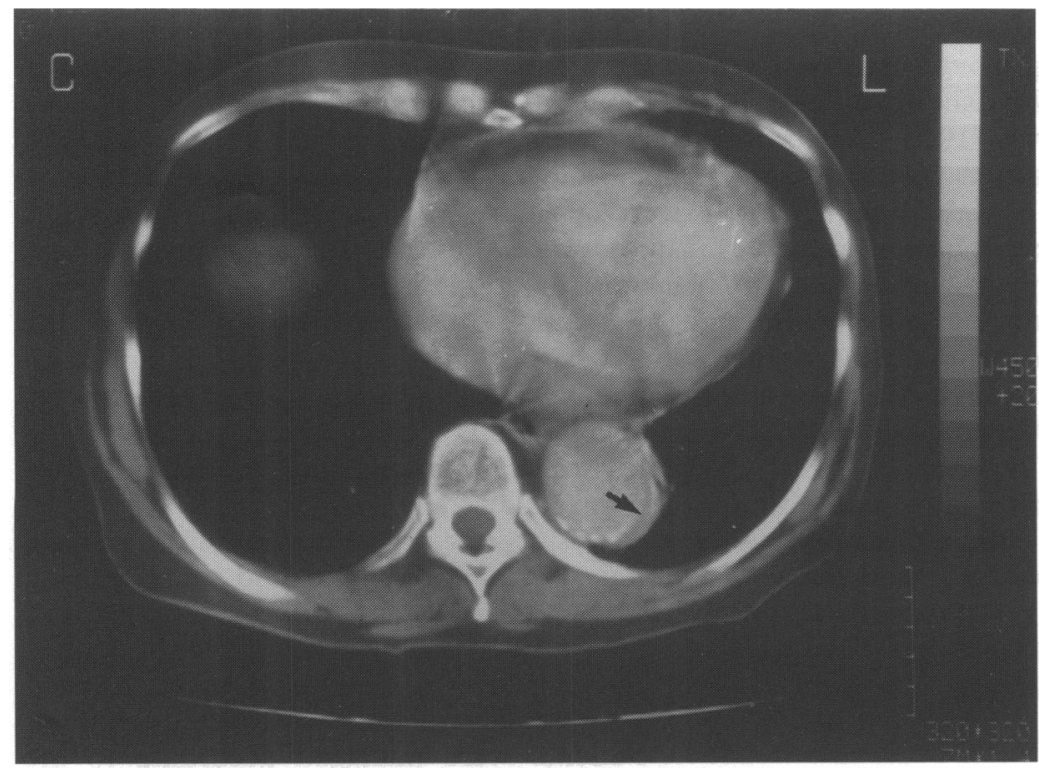

Figure 1 Computed tomography scan of a thorax reported as showing a dilated aortic root and possible descending aortic dissection (arrow).
Eight patients with type A dissection had pericardial effusions and in three patients this was associated with echocardiographic features of tamponade. Transoesophageal echocardiography showed moderate aortic regurgitation (origin of the regurgitant jet on colour M Mode $25-50 \%$ of left ventricular outflow diameter) in six patients and severe aortic regurgitation (regurgitant jet $>50 \%$ of left ventricular outflow tract diameter) in seven. The origin of the left coronary artery was identified in all patients and the origin of the right in $17 / 22(77 \%)$.

Twenty three of the 48 patients (48\%) had no evidence of aortic dissection on transoesophageal echocardiography. Atheroma of the thoracic aorta was noted in nine, coarctation of the aorta in one, and hypertrophic cardiomyopathy in one patient.

No patient without evidence of dissection on initial transoesophageal echocardiography underwent further investigation into possible dissection. One of these patients died in hospital of a myocardial infarction, which was confirmed at necropsy. The others were discharged. The final discharge diagnoses were ischaemic heart disease in 11 , musculoskeletal chest pain in seven, and non-specific chest pain in five.

OTHER DIAGNOSTIC INVESTIGATIONS AFTER TRANSOESOPHAGEAL ECHOCARDIOGRAPHY

After transoesophageal echocardiography two patients underwent further diagnostic investigations.

One patient with type $\mathrm{A}$ dissection and a history of previous myocardial infarction underwent coronary angiography, but because of procedural difficulties neither coronary artery was shown selectively. Graft replacement of the ascending aorta (without a coronary bypass operation) was performed successfully.

A patient with a long history of intermittent chest and back pain had evidence of a thrombosed type A dissection on transoesophageal echocardiography. Computed tomography was performed to confirm the findings in the upper ascending aorta; she was managed medically and discharged home.

INVESTIGATIONS BEFORE TRANSOESOPHAGEAL ECHOCARDIOGRAPHY

Sixteen patients had undergone computed tomography before referral to the regional centre. On arrival three were unfit for an operation or transoesophageal echocardiography and therefore 13 underwent transoesophageal echocardiography in the cardiac care unit The diagnoses and classification of dissection were confirmed in seven patients. In two patients transoesophageal echocardiography showed involvement of the ascending aorta and arch, which had not been visualised by computed tomography (figs 1 and 2). This reclassification altered management in the two patients.

Four patients referred after computed tomography thought to show dissection had no evidence of dissection on transoesophageal 


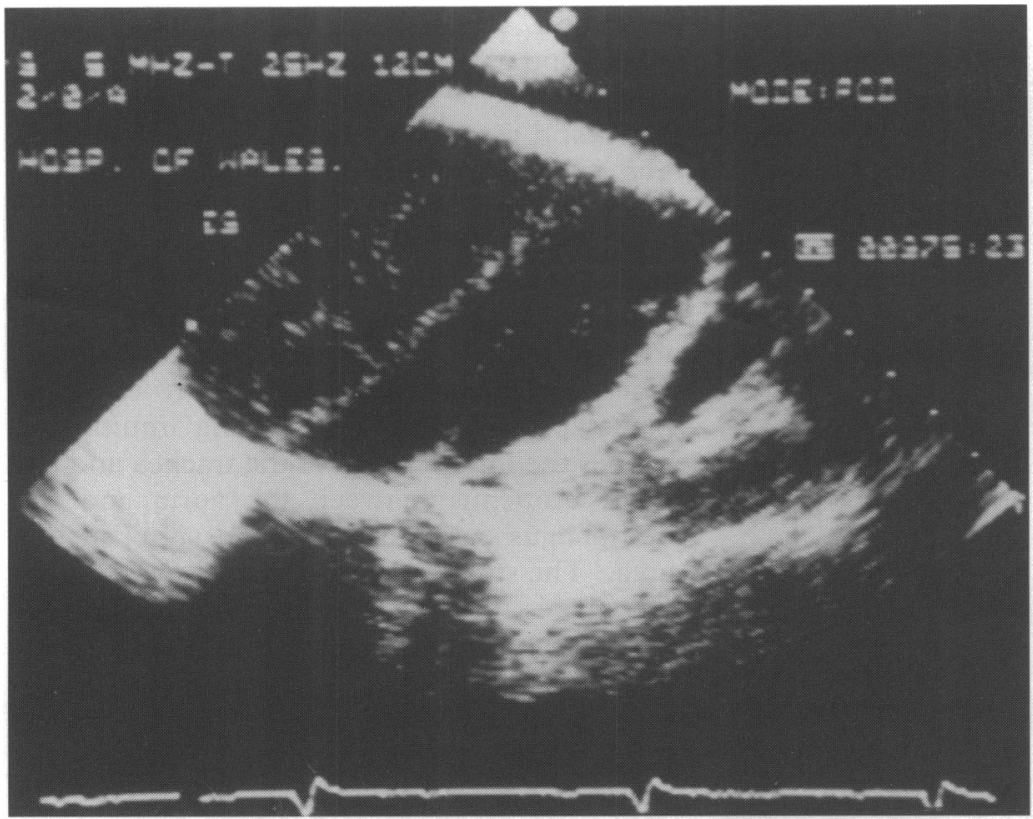

Figure 2 Transverse two dimensional transoesophageal echocardiogram in the same patient showing a spiral dissection of the ascending aorta and spontaneous echo contrast in the false lumen. The descending aorta was atheromatous and heavily calcified with no evidence of dissection.

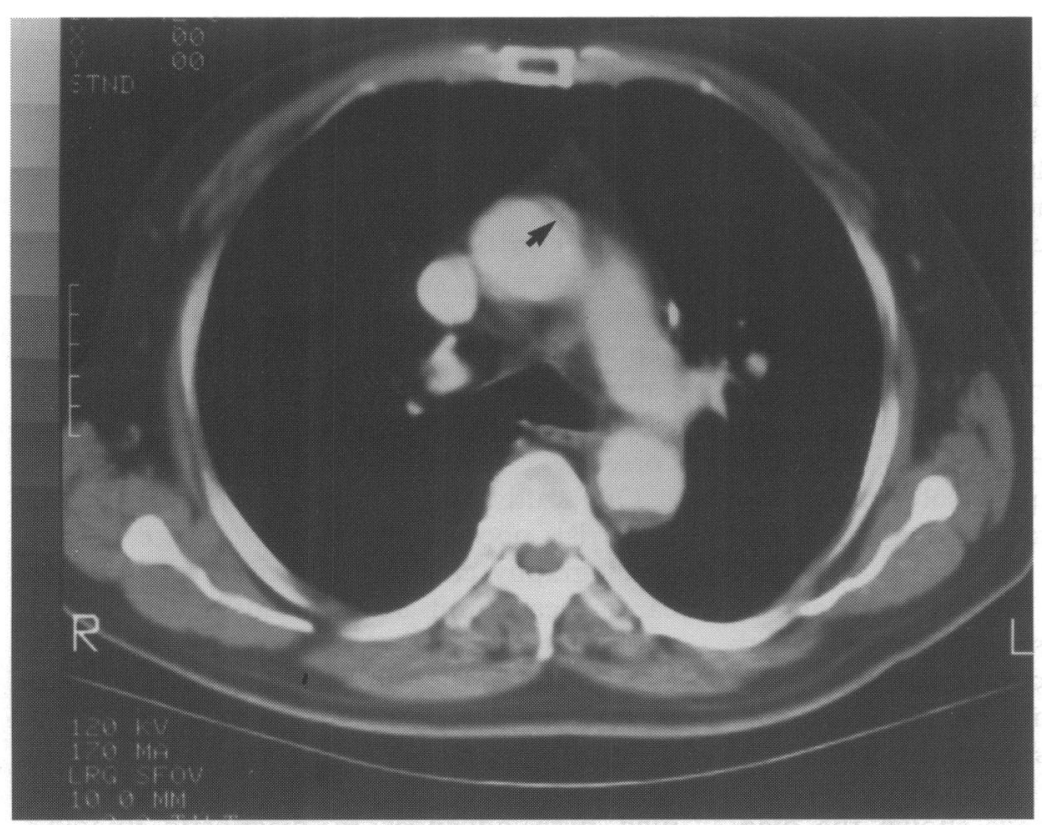

Figure 3 Contrast enhanced computed tomography scan of the thorax suggesting dissection of the ascending aorta (arrow). in theatre was 2.5 hours. In all patients the ascending aorta was replaced with a dacron graft. The aortic valve was resuspended in two patients and replaced in three. One patient had coronary artery reimplantation.

One patient died post-operatively after developing refractory ventricular fibrillation. Autopsy revealed trivial coronary disease, no evidence of recent myocardial infarction, and confirmed dissection of the aorta. Nine patients who had an operation were discharged from hospital.

Five patients with type A dissections were felt to be unfit for an operation; each had evidence of renal dysfunction, limb ischaemia, and impaired consciousness. All died within 24 hours of admission and in all dissection was confirmed at necropsy.

Two patients with type A dissection were electively managed medically. One patient was felt to be too elderly for surgery (aged 83 years) and he was discharged home after medical treatment. A second patient with a chronic type A thrombosed dissection was managed conservatively and was discharged.

Dissection of the descending aorta (type B)

Two patients with type $B$ dissection developed evidence of mesenteric ischaemia; one was judged to be unfit for an operation and died (autopsy confirmed the diagnosis) and the second underwent bowel resection but died post-operatively (autopsy confirmed thoraco-abdominal aortic dissection with evidence of renal and mesenteric infarction). Six of the eight patients with type B dissection were discharged home.

\section{HOSPITAL OUTCOME AND LONG TERM FOLLOW} UP

The hospital survival for all patients with type A dissection was $8 / 17(47 \%)$. This included 9/10 (90\%) patients who had an operation and $2 / 7(29 \%)$ patients treated medically. At a mean of two year follow up $8 / 9$ surgical patients were alive, one having died 23 months after discharge from a stroke. Seven were fit and well with no subsequent hospital admissions. One had a dense hemiplegia which had persisted since the operation. Of the two patients with type A dissection treated medically, one died suddenly 24 months after discharge (no necropsy) and the other was alive and well.

The hospital survival for all patients with type B dissection was $6 / 8$ (75\%). At a mean of two years follow up, four were alive and well, and one patient was being investigated for unexplained syncope. One patient died 17 months after discharge (no necropsy).

Follow up information was available in all 22 patients who were discharged from hospital and had no evidence of dissection on transoesophageal echocardiography. All were alive two years later (mean follow up 32 months, range 24-44 months). Seven had been readmitted to hospital in the intervening period; four had had myocardial infarcts (documented by electrocardiographic and cardiac enzyme changes), two patients with severe eight and within 24 hours in two patients. The mean delay in patients having an immediate operation from arrival in the hospital to arrival 


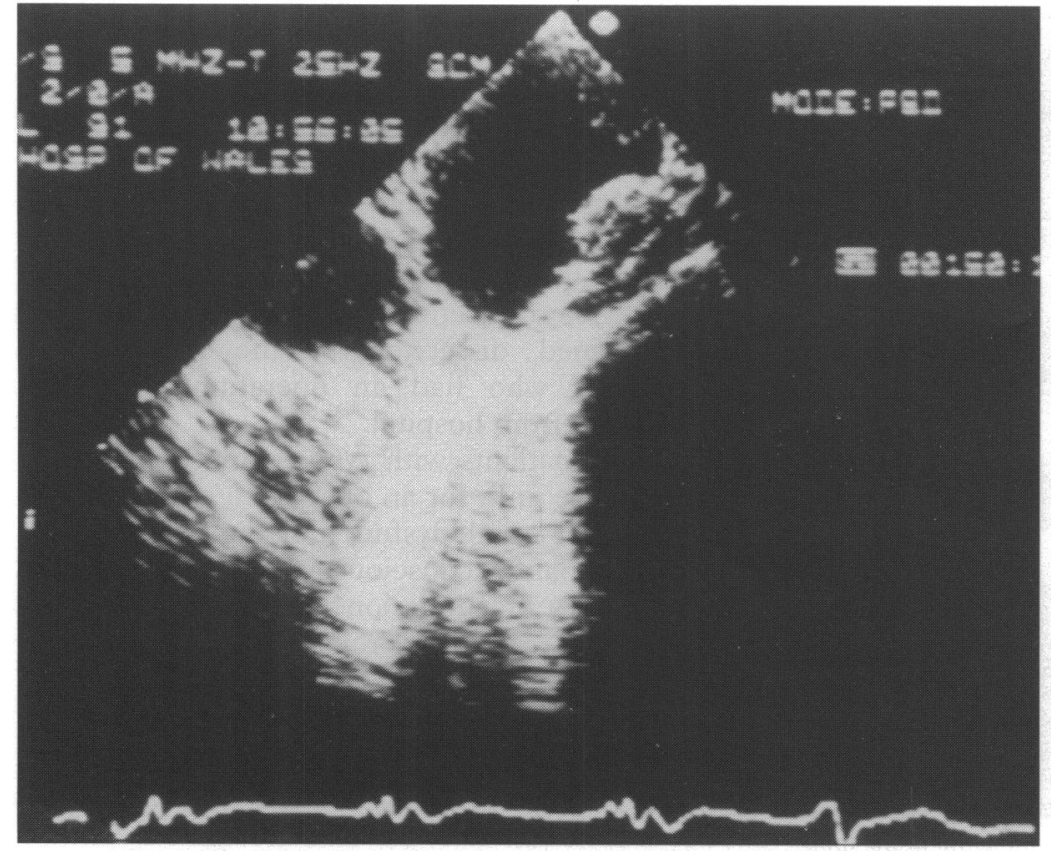

Figure 4 Two dimensional transoesophageal echocardiogram in the same patient showing calcified atheroma and intraluminal thrombus, but no evidence of aortic dissection.

aortic regurgitation had had an aortic valve replacement, one patient with coarctation of the aorta had had a balloon dilatation, and one patient had been admitted with recurrent atypical chest pain. None had required repeat investigation for possible dissection.

\section{Discussion}

The pivotal role of an immediate operation in patients with dissection of the ascending thoracic aorta is now established. Patients with suspected dissection require safe, rapid, and accurate imaging to provide diagnostic and prognostic information. ${ }^{1}$

Angiography has been used as the diagnostic method of choice for assessing patients with suspected thoracic dissection since $1945.5^{5}$ For many years it was the only investigation available, but comparative series with computed tomography, magnetic resonance imaging, and echocardiography have shown the diagnostic superiority of these non-invasive techniques. $^{2}$

Before undertaking an operation, the surgeon must know the entry site of the dissection and the degree to which the ascending aorta is affected. The decision to operate and the type of operation to be undertaken will be influenced by the competence of the aortic valve, the presence of pericardial effusion/ tamponade, and whether the coronary arteries are affected. This information must be obtained in the minimum time as untreated thoracic aortic dissection carries a mortality of at least $1 \%$ /hour. ${ }^{6}$

Our unit has selected transoesophageal echocardiography as the primary diagnostic imaging modality in suspected dissection. It is able to give detailed anatomical information about the morphology of a dissection and it can also show the consequences of proximal extension. It can be performed rapidly by a single operator with nursing assistance, in an environment where the patient can be monitored and remains accessible to medical staff, and is therefore an ideal diagnostic tool for emergencies.

The use of transoesophageal echocardiography in dissection has been criticised as imaging of the upper ascending aorta is limited and there is a high incidence of artefact. ${ }^{7}$ These problems apply particularly to single plane probes, but even using multiplane probes the interposition of the trachea and left main bronchus between the aorta and the oesophagus can cause a "blind spot" in this area. ${ }^{2}$ The suprasinus ridge just above the aortic valve is the most common site of a dissection tear in the ascending aorta. ${ }^{8}$ Visualisation of this area by transoesophageal echocardiography is excellent and our series shows that in most patients transoesophageal echocardiography alone gives sufficient information to guide patient management.

Echocardiographic artefact can occur on transoesophageal echocardiography in up to $40 \%$ of patients with dilated aortic roots. ${ }^{9}$ Recognition of this possibility and the use of $M$ mode and colour flow Doppler ultrasound has been shown to distinguish correctly $99 \%$ of cases. ${ }^{9}$ The single occurrence false positive transoesophageal echocardiography in our series was in a patient who had a definite dissection of the descending aorta and a $7.5 \mathrm{~cm}$ diameter aortic root. The linear shadow in the ascending aorta was related by artefact to aortic wall calcification. He underwent emergency root replacement which, although "appropriate", could have been carried out electively if the artefact had been recognised correctly.

Our study also confirms that even in this high risk population transoesophageal echocardiography can be performed safely in the cardiac care unit using intravenous sedation and antihypertensive drugs. This finding is consistent with a published complication rate of less than $1 \% .^{3}$

Improvements in surgical and anaesthetic techniques over the last 15 to 20 years, coupled with advances in diagnostic imaging, have reduced the mortality from thoracic aortic dissection. ${ }^{10}$ Excision of the intimal tear in the ascending aorta with interposition of a dacron graft is the most commonly performed procedure. ${ }^{11}$ In general, replacement of the aortic valve is only performed when resuspension of the valve by supporting the valve commissures is impossible. Attitudes about performing bypass surgery for "coincidental" coronary disease at the same time as emergency aortic surgery are changing, because in unstable patients the prolongation of cardiopulmonary bypass time may increase perioperative mortality. When coronary surgery is not contemplated, coronary angiography is only necessary to define whether the coronary ostia are affected. If this information is obtained by transoesophageal echocardiography, preoperative cardiac catheterisation is no longer justified. 
Computed tomography has been used as a diagnostic technique in dissection for over 10 years. ${ }^{12}$ It is minimally invasive and is increasingly available in district general hospitals. Studies from specialist centres have shown a sensitivity of 82 to $100 \%$, with a specificity of between 90 to $100 \% .^{13}$ In practice, good quality high density computed tomographic images can be difficult to obtain in an emergency and poor images increase the incidence of artefact and false positive diagnoses. Transoesophageal echocardiography on arrival in our centre, performed after computed tomography at a district general hospital, altered the classification and management in $38 \%$ of patients. This represents not only the limitations of interpretation of computed tomography data by radiologists and doctors who have limited experience in thoracic aortic imaging, but also a tendency to "over-report" abnormalities to ensure that the diagnosis is not missed.

Performing computed tomography before referral to a regional centre significantly increases delay. This delay occurs during the period of maximum mortality, and in our experience the diagnostic information obtained is unreliable. As transoesophageal echocardiography is currently not available in the local district general hospitals, we now recommend immediate referral of patients with a high clinical index of suspicion of dissection for diagnostic imaging and management. This approach aims to minimise preoperative delay, which is fundamental to further reductions in the mortality from acute thoracic aortic dissection.

\section{Study limitations}

This study was designed to assess the efficacy of using transoesophageal echocardiography alone as a diagnostic modality in aortic dissection. The high diagnostic sensitivity of transoesophageal echocardiography is well documented, ${ }^{2314}$ but it is possible that the investigation may not have detected dissection in some patients and that any such dissections have since remained occult. The excellent clinical outcome of these patients with "negative" results suggests that evidence of dissection was not missed by transoesophageal echocardiography. Diagnostic certainty could have been increased by using another imaging technique such as magnetic resonance imaging, but in the context of this study this was inappropriate unless clinically indicated.
Patients included in this study were identified retrospectively from five separate sources. As the University Hospital of Wales is the only cardiothoracic surgical centre in Wales, it is likely that this series represents a complete sample of all patients with possible dissection referred for a surgical opinion from the surrounding hospitals during the period of the study. The study was not designed to assess either the true incidence of dissection in the community or its in hospital mortality.

\section{Conclusions}

Our experience shows that transoesophageal echocardiography provides all the diagnostic information required to make management decisions in patients with a suspected dissection of the aorta. Further investigations, including coronary angiography before operating, are unnecessary and emergency aortic surgery can be performed rapidly and successfully based on transoesophageal echocardiography findings alone.

The authors acknowledge the assistance of colleagues and staff of the cardiac unit at the University Hospital of Wales. APB is supported by a BHF Junior Research Fellowship.

1 Treasure T. Imaging the dissecting aorta. Br Heart $\mathcal{f} 1993$; 70:497-8.

2 Khandheria BK. Aortic dissection; the last frontier. Circulation 1993;87:1765-8.

3 Erbel R, Engberding R, Daniel W, et al. Echocardiography in diagnosis of aortic dissection. Lancet 1989;i:457-61.

4 Daily PO, Trueblood HW, Stinson EB, Weurflein RD, Shumway NE. Management of act
Ann Thorac Surg 1970;10:237-47.

5 Clague J, Magee P, Mills P. Diagnostic techniques in suspected thoracic aortic dissection. Br Heart $\mathcal{f} 1992 ; 67$ : pected

6 Harris PD, Malm JR. The management of acute dissection of the thoracic aorta. Am Heart $\mathcal{F}$ 1969;78:419-22.

7 Reid JH, Gillespie IN. Imaging methods used in aortic dissection [letter]. BMF 1994;308:535-6.

8 Dalen JE, Pape LA, Cohn LH, Koster K, Collins JJ. Dissection of the aorta: pathogenesis, diagnosis and treatment. Prog Cardiovasc Dis 1980;23:237-45.

9 Evangelista A, Gonzalez-Alujas T, Garcia del Castillo $H$, Anivarro I, Salas J, Soler-Soler J. Are artefacts a major drawback for the diagnosis of ascending aortic dissection by transoesophageal echocardiography [abstract]. Eur Heart $\mathcal{f}$ 1993;14(suppl):391.

10 Chirillo F, Marchiori L, Andriolo L, et al. Outcome of 290 patients with aortic dissection. A 12-year multicentre

11 Taylor KM. Diseases of the aorta. In: Julian DG, Camm AJ, Fox KM, Hall RJC, Poole-Wilson PA, eds. Diseases of the Heart. London: Balliere Tindall, 1989:1348-62.

12 Cigarroa JE, Isselbacher EM, Desanctis RW, Eagle KA. Diagnostic imaging in the evaluation of suspected thoracic aortic dissection. $N$ Engl f Med 1993;328:35-43.

13 Nienaber CA, von Kodolitsch Y, Nicolas V, et al. The diagnosis of thoracic aortic dissection by noninvasive imaging procedures. $N$ Engl $₹$ Med 1993;328:1-9.

14 Ballal RS, Nanda NC, Gatewood R, et al. Usefulness of transoesophageal echocardiography in the assessment of aortic dissection. Circulation 1991;84:1903-14. 